Human monkeypox virus infection. The recent outbreak of Human Monkeypox Virus disease in Midwestern States and the governmental decision to recommend smallpox vaccination to persons exposed to prairie dogs or Gambian giant rats, pets linked to the infection, provide neurologists with an additional reason to consider the implications of smallpox vaccination. Human monkeypox is a rare zoonotic viral disease that occurs primarily in the rain forest of central and west Africa. Person-to-person spread may occur. The incubation period is up to 21 days. Fatalities in Africa have ranged from one percent to $10 \%$ of cases. Most patients seen in the US have experienced a prodrome of fever, headaches, myalgia, chills and sweats, followed by a nonproductive cough in one-third. A papular rash developed at one to 10 days after onset of fever and it progressed to vesiculation, pustulation, umbilication and crusting. Lesions occurred on head, trunk and extremities, including palms and soles. In the US, among 22 cases contracted from pet prairie dogs no fatalities have been reported. (Conover CS, Illinois Department of Public Health, Springfield, IL, June 8, 2003).

\title{
NEUROLOGIC COMPLICATIONS OF ANTHRAX
}

The literature on neurologic complications of infection by Bacillus anthacis is reviewed at the Dent Neurologic Institute, Buffalo, NY. The major neurologic complication is a rapidly fatal hemorrhagic meningoencephalitis. The initial mode of entry of the bacillus is via the cutaneous or inhalation route. One of the earliest cases of hemorrhagic meningoencephalitis from anthrax, in a boy aged 15 years, was characterized by numerous focal bleeds in the surface grey matter but very few hemorrhages in the white matter (House SJ. J Infect Dis 1920;27:513-526). House also reported autopsies on 2 adults with anthrax-related hemorrhagic meningitis, contracted in a Chicago factory by handling "curled hair" from South America. Other cases have involved contaminated heroin from Afghanistan, Pakistan, and Iran, in drug users, contaminated beef in slaughter house workers, and contact with sheep in farmers. CSF findings in 2 children with anthrax meningitis showed low glucose, increased protein and leucocytes, and large gram-positive rods without endospores on gram stain. CT scan shows multifocal areas of intracerebral hemorrhage. Inhalation cases of anthrax meningoencephalitis, affecting half the cases autopsied in the Russian 1979 outbreak, are particularly severe. In a recent report of fatal inhalation anthrax due to bioterrorism, the patient developed a generalized seizure within hours of admission and died on the third hospital day (Bush LM et al. New Engl I Med 2001;345:1607-1610). Death usually occurs within a week in cases of anthrax with neurologic involvement. Further research is needed to develop more rapid PCR methods to detect the disease in suspected cases. Antibiotic treatment with ciprofloxacin or doxycycline must be started immediately when the diagnosis is first apparent. (Meyer MA. Neurologic complications of anthrax. A review of the literature. Arch Neurol April 2003;60:483-488). (Reprints: Michel A Meyer MD, Dent Neurologic Institute, 3980 Sheridan Dr, Amherst, NY 14226).

COMMENT. Anthrax should be considered in the differential diagnosis in a patient with fever, dark necrotic pustules on the extremities, acute neurologic deterioration, grampositive rods in the CSF, and multifocal intracerebral hemorrhages on CT. 